\title{
7xxx aluminum alloys; strengthening mechanisms and heat treatment: a review
}

\begin{abstract}
The intention of authoring this paper was giving the report of reviewing task of the previous researches done on the strengthening mechanisms and heat treatment of $7 \mathrm{xxx}$ aluminum alloys. The overall conclusion is that, the most powerful and so the most common strengthening mechanism in $7 \mathrm{xxx}$ alloys is the precipitation hardening (T6 heat treatment). On the other hand, stress corrosion is a problem in T6 treated $7 \mathrm{xxx}$ alloys, to address which, researchers have turned to special heat treatment called RRA (retrogression and re-aging) which picks the strength from T6, and stress corrosion resistance from $\mathrm{T} 7 \mathrm{x}$ heat treatments.
\end{abstract}

Keywords: aluminum alloys, AA7xxx, strengthening, heat treatment, RRA
Volume 2 Issue 2 - 2018

\author{
Amir Jodeiri Feizi, Mehrdad Ashjari \\ University of Tabriz, Iran
}

Correspondence: Mehrdad Ashjari, University of Tabriz, Iran, Tel +989|430995 I5, Email Mhd_ashjari@yahoo.com, Mehrdad_ dts9l@Ms.tabrizu.ac.ir

Received: February 16, 2018 | Published: April 20, 2018

\section{Introduction}

The earth is being depleted of its natural resources, and it is of absolute importance to retard this destroying trend, which is for engineers to do so. One of the ways of saving the perishing environment is finding ways to contribute towards less energy consumption. One among many of these contributing methods is making fossil fuel killers, e.g. cars, airplanes, etc. lighter which is possible through using lighter metals like aluminum in their structure. One limitation in using a pure light metal may be its lack of strength. But the goal is worth fighting all the obstacles, and this fight is at least as old as the existence of blacksmith who beat a piece of metal to render it stronger. Among likely candidates for light structure material, is aluminum, to which because of its unique properties, to mention one among many, not showing brittle response under an specific temperature (transition temperature) in contrast to steel, ${ }^{1}$ which makes it an ideal metal for cryogenic conditions, industry and as a result research, are showing greater interest. The good thing about this element is its high strength to weight ratio into the bargain; ${ }^{2}$ the drawback is its relatively low strength. These two characteristics make aluminum suitable for low stress applications. But most of engineering purposes deal with moderate to high stresses in which pure aluminum may give in. To overcome this, it is an old fashion, as one of the methods of enhancing this metal, to add other elements like copper, manganese, silicon, magnesium, zinc, etc. to it as alloying elements. In addition to alloying elements in the base metal there exist some other interesting strengthening methods such as, microstructure modification (grain size reduction), precipitation hardening, dispersion strengthening, and work hardening. One or combination of these may be applied to alloy depending on its properties and limitations. It seems, from among alloying elements, zinc and magnesium play greater role in giving the aluminum strength. $7 \mathrm{xxx}$ aluminum alloys which contain zinc and magnesium, as their main alloying elements in varying amounts (which define the rest of the digits after number " 7 " in the name of the alloy) have gained the top priority when considering nominees for suitable material for aero-transportation vehicles. For, in the airplane, safety is the main issue, in addition to strength of components, other factors like stress corrosion must be taken into account at the time of designing the components.
All strengthening mechanisms rely on one principle; Hindering movement of dislocations. All existing methods like Strain hardening, ${ }^{3}$ precipitation hardening, ${ }^{4,5}$ Hall-Petch effect (grain boundary strengthening) ${ }^{6,7}$ dispersion strengthening, ${ }^{8}$ make the alloy stronger by decreasing the degree of mobility of the dislocations. Talking about aluminum, depending on the major alloying element, the contribution of each of the mechanisms differ dramatically. For example, if an aluminum alloy has the copper as its major alloying element (2xxx series of wrought aluminum alloy) it can be precipitation hardened; on the other hand, if copper is superseded by magnesium ( $5 \mathrm{xxx}$ series of wrought aluminum alloy), precipitation strengthening will have negligible contribution towards the yield strength augmentation of alloy. $7 \mathrm{xxx}$ aluminum alloys, are called heat treatable alloys as they can be strengthened through precipitation strengthening. One of the notorious shortcomings of precipitation hardened $7 \mathrm{xxx}$ alloys is their lack of resistance against stress and exfoliation corrosion. Industry owners and researchers have turned to a special heat treatment called retrogression and re aging to make the stress corrosion to surrender by a great degree.

\section{Literature and discussion}

Kaka Ma et al.,9 investigated the strengthening mechanisms of UFG (ultrafine grained) and CG (coarse grained) AA7075 in both extruded and T6 tempered conditions. Their research revealed that, the dominant strengthening mechanism in as extruded UFG is the Hall-Petch effect with a magnitude of contribution of $242 \mathrm{Mpa}$; while for the same structure (UFG) in T6 tempered condition, the dominant contributing mechanism is the Orowan mechanism which increased the strength by $414 \mathrm{Mpa}$. They also report that, in CG AA7075, for both as extruded condition as well as T6 tempered condition, the dominant strengthening mechanism is the Orowan mechanism, with contribution of $103 \mathrm{Mpa}$ and $472 \mathrm{Mpa}$ respectively. Zhang et al., ${ }^{10}$ studied the effects of confined channel die pressing (CCDP) which is one of SPD (superplastic deformation) methods, on an aluminum alloy belonging to 7000 series, and reported that, resultant microstructure is UFG one with low angle subgrains with $100-200 \mathrm{~nm}$ of diameter. Figure 1 show the way the mechanical properties of the alloy benefited from CCDP. As the stress-strain curves indicate, both tensile strength 
and elongation is minimum for the base metal (F0; which is the alloy before SPD), and with increasing number CCDP passes, from 1 to 18 (F1 F18) the tensile strength increases.

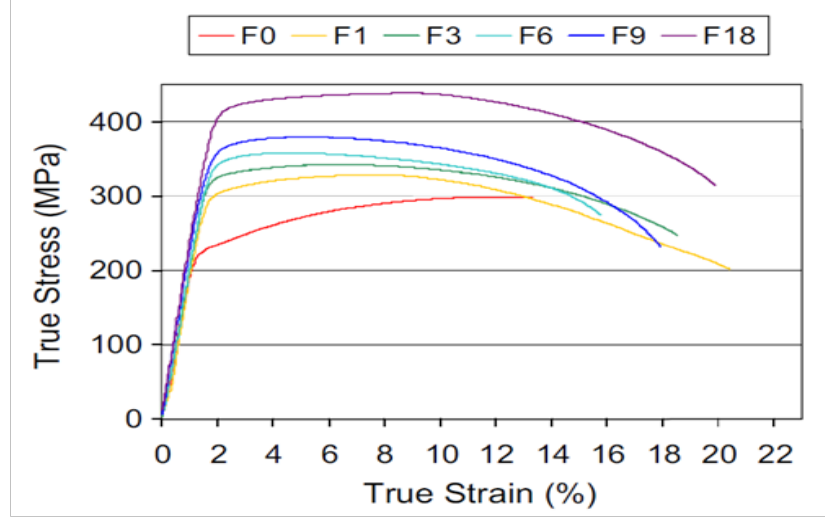

Figure I Stress-Strain curves of the samples after various numbers of CCDP passes. ${ }^{10}$

Xiaofeng Xu et al., ${ }^{11}$ investigated the effect of Ag addition on the mechanical properties of the precipitation hardened $7075 \mathrm{Al}$ alloy. They revealed that addition of $\mathrm{Ag}$ (as much as $0.4 \mathrm{wt} \%$ ) not only increased the YS and UTS by $25 \mathrm{Mpa}$ (from 425 to 447) and $27 \mathrm{Mpa}$ (from 528 to 555) respectively, but also decreased the precipitation process time to get the peak hardness by $54 \%$. They attribute this enhancement in mechanical properties to the precipitation of $\Omega$ which possesses less coarsening rate and better strengthening effect in comparison with $\theta^{\prime}$ phase. Junhou et al., ${ }^{12}$ investigated the mechanical behavior of 7055 aluminum alloy aged at two different temperatures, $120^{\circ} \mathrm{C}$ and $160^{\circ} \mathrm{C}$. The report of their research reveal, for the alloy aged at $120^{\circ} \mathrm{C}$, yield strength increases to a peak value and the remains stable with the aging time. On the other hand the alloy aged at $160{ }^{\circ} \mathrm{C}$, the strength decreases after it reaches a peak value as the aging time increases. The increasing trend of the yield strength before it reach a peak value, is because as the aging time increases the volume fraction and size of the y' increases. But then, for the sample aged at the $120^{\circ} \mathrm{C}$, the size and the volume fraction of the y' phase remains approximately constant, whereas in the sample aged at $160{ }^{\circ} \mathrm{C}, \mathrm{y}$ forms and coarsens as the aging time goes on, which transforms the precipitate-dislocation interaction from cutting to looping and causes the strength to gradually drop with time. In a research done by Shengdan et al., ${ }^{13}$ effects of existing quench induced precipitation in the microstructure of 7085 aluminum alloy on the mechanical behavior of this alloy was clarified. They studied the mechanical behavior of naturally and artificially precipitated AA7085 in two conditions: one high rate cooling condition $(240 \mathrm{~K} / \mathrm{s})$ and low rate cooling condition $(3.2 \mathrm{~K} / \mathrm{s})$. Their report contains interesting findings. It is generally believed that, because high cooling rate causes high solute atom density after quenching, the final microstructure will possess higher mechanical strength because of higher y' phase precipitation. Interestingly, fast quenched-naturally aged alloy has 37 Mpa less of yield strength comparing to slow quenched-naturally aged alloy. This higher strength is attributed to the existence of Y phase which owns high aspect ratio and precipitates in $\{111\}$ plane. In the naturally aged specimen, the strength drops with lower cooling rate as expected. In the slow quenched specimen, some quench-induced precipitate $\mathrm{y}$ occurs and depletes the microstructure with $\mathrm{Zn}$ and $\mathrm{Mg}$, so the amount of strengthening y' phase decreases after aging, which causes the specimen have less strength than the fast-cooled one. To better compare the effect of the cooling rate on the naturally as well as artificially aged specimen, the yield strength and hardness of the specimen are shown in Table 1.

Table I Effect of the speed of cooling in quenching on the mechanical properties of specimen 13

\begin{tabular}{llll}
\hline Cooling rate & Aging & Yield strength & Hardness \\
\hline $3.2 \mathrm{~K} / \mathrm{s}$ & Naturally aged & 303 & 121.9 \\
$240 \mathrm{~K} / \mathrm{s}$ & Naturally aged & 266 & 114.5 \\
$3.2 \mathrm{~K} / \mathrm{s}$ & Artificially aged & 485 & 178.4 \\
& & & 190.3 \\
\hline
\end{tabular}

To avoid the distortion and oxidation that are inherent to most of heat treatments including solid solution heat treatment, Xiaofeng et al. ${ }^{14}$ made use of electropulsing treatment (EPT) to make supersaturated solid solution and they compared it with traditional solid-solution treatment (SST). As they report, although the degree of dissolution in EPT is lower comparing with SST, for the smaller grain size of the microstructure of the EPTed alloy, an alloy with more strength and slightly less ductility is obtained after EPT and artificial aging. Also, no distortion and oxidation happens in the EPT+AA alloy. Xiao $\mathrm{Wu}$ Nie et al., ${ }^{15}$ conducted experimental study on the double-peak precipitation hardening and strengthening mechanism of $7 \mathrm{xxx}$ alloy. They found, regarding the strengthening mechanism, shearing the strengthening particles and precipitates transforms into looping them in the early stages of particle growth and that looping is the dominant strengthening mechanism. They also developed a model to simulate the kinetics of precipitation and its predictions about hardness matched well with the experimental value. Wenjing Yang et al., ${ }^{16}$ performed double sided friction stir processing on the 7050-T7451 aluminum alloy. Their findings show that, DFSP not only eliminated the TMAZ and HAZ regions, which are pernicious to the mechanical properties of the processed area, but also caused drastic grain refinement in the area. Alvandi et al., ${ }^{17}$ investigated the mechanical behavior of accumulative roll bounded (ARBed), AA7075. Their finding clarified that, after 6 cycles of ARB the microstructure possesses grains of 130 $\mathrm{nm}$ averagely which is about $36 \mu \mathrm{m}$ for the alloy before ARB process. This noticeable reduction in grain size, causes the UTS to increase by 3 times after ARB. Yuguo Liao et al., ${ }^{18}$ investigated the effect of $\mathrm{Cu}$ addition on the mechanical behavior of $7 x x x$ series aluminum alloy. In their paper, they report that the dominating strengthening phase is y' phase which increases with copper content in the alloy. Also, increase of $\mathrm{Cu}$ from 0 to $1.6 \%$ causes an enhancement of tensile strength by 59 Mpa, from $515 \mathrm{Mpa}$ to $574 \mathrm{Mpa}$. Jinrong Ju et al., ${ }^{19}$ in a paper reporting the findings of their research on the effect of double step hot rolling (DR) on the mechanical properties of 7055 aluminum alloy, revealed that, DR caused alloy possess high toughness. DR by generating fine $\mathrm{MgZn}_{2}$ particles which pin grain boundaries, prevent grains from getting coarse. Lavernia et al., ${ }^{20}$, classified the aluminum alloys into 3 categories; in addition to well known heat treatable and non-heat treatable alloys, the third type of alloys are the $7 \mathrm{xxx}$ aluminum alloys with zirconium, nickel, cesium and iron ingredients which by lowering the solubility and diffusivity of solute in the matrix, hinders the enlargement of the precipitates, and so increases thermal stability of the alloys. Also, it is stated that, cobalt addition (0.4-1.5 wt \%) in $7 \mathrm{xxx}$ 
alloys, works as grain size stabilizer. Seungwon Lee et al., ${ }^{21}$ looked into the possibility of simultaneously applying two strengthening methods, grain refining and precipitation, on AA7075 through HPS (high pressure sliding) method which is one of several severe plastic deformation methods, and studied the effect of such strengthening operation on the mechanical properties of the alloy. In their paper it is reported that, simultaneous strengthening has favorable effect on the mechanical properties of 7075 aluminum alloy, and the UTS as well as the YS increased after HPS and aging treatment. Also the grain size dropped to a value around $280 \mathrm{~nm}$. One of the main obstacles concerning the precipitation hardening (T6) of 7000 aluminum alloys, is decreasing the stress corrosion cracking (SCC) resistance of the alloy. ${ }^{21}$ To increase the mechanical strength of the alloy and at the same time maintain the SCC resistance of the alloy, Fooladfar et al., ${ }^{22}$ made use of shot-peening before conducting T6 heat treatment. One of the affecting items on the SCC is the size and the distance between the grain boundary precipitates (GBP) and it is stated that increasing the size and distance between GBPs, in other words, over-aging the GBPs is the remedy for SCC being decreased after T6. Instead of manipulating the GPBs in bulk scale, for the surface of the part is most susceptible against SCC, it seems logical to work on the outer layer of the part. On the premise that, dislocations in the microstructure, play the role of sites suitable for heterogeneous direct nucleation of the $\mathrm{y}$ precipitates, any practice, like plastic deformation, resulting in the creation of dislocations, causes the microstructure become more talented in getting over-aged after precipitation treatment. It seems that, shot-peening besides all well-known effects, because of the plastic deformation it causes, may have the ability to overage the GBPs of the surface of the part after precipitation treatment. To verify this, Fooladfar ${ }^{22}$ applied the explained procedure to the 7075 aluminum alloy and investigated the effects on the SCC behavior as well as the mechanical behavior of the alloy. Their study revealed that, shot peened then T6 heat treated alloy has both less SCC susceptibility and the same mechanical properties as $\mathrm{T} 6$ alloy. There exist numerous literature addressing the $\mathrm{SCC}$ problem in $7 \mathrm{xxx}$ alloys via retrogression and re-aging (RRA) method. ${ }^{23-27}$ Gokhan Ozer et al., ${ }^{28}$ made use of RRA method to increase the resistance of the AA7075-T6 against SCC or revive the lost mechanical strength in T73 treated alloy. They particularly investigated the effect of the temperature and the duration of retrogression and re aging heat treatment on the mechanical and physical behavior of AA7075. They reported that, increasing the retrogression period, the hardness of the alloy decreases. The highest hardness value is obtained at RRA treatment at $180{ }^{\circ} \mathrm{C}$ for 4 mins. Also, all the RRAed samples, regardless of the time and duration of the treatment, showed higher electrical conductivity, as the findings of their research say. LI Guo-feng et al., ${ }^{29}$ conducted research to find out the influence of heating speed in retrogression step on the mechanical strength of AA7050. They suggest that, heating rate has a significant effect on the state of strengthening precipitates (GP and n') after reaging step, and so is influential in defining the mechanical properties of the alloy. Retrogression was conducted in three different heating up speeds, $4.3{ }^{\circ} \mathrm{C} / \mathrm{min}$ (low speed), $57{ }^{\circ} \mathrm{C} / \mathrm{min}$ (medium speed) and $340{ }^{\circ} \mathrm{C} / \mathrm{min}$ (high speed). The resultant mechanical strength is the highest after RRA with medium speed of heating in retrogression step. In low speed mode, because there exist sufficient time for phase transformation, $\mathrm{y}$ ' transforms into $\mathrm{y}$ phase, and as a result of $\mathrm{\eta}$ phase coarsening, the mechanical strength drops. In contrast to high speed mode, in medium heating speed, re-precipitation of GP and y' is completed after re aging step, and as a result RAA with medium speed of heating up in retrogression step renders the alloy strongest comparing to two other heating rates. As Figure 2 shows, the hardness is the highest in medium speed mode. By the passage of the time the hardness value decreases, and this is the trend to a level where it reaches the same value as the hardness of the high speed mode.

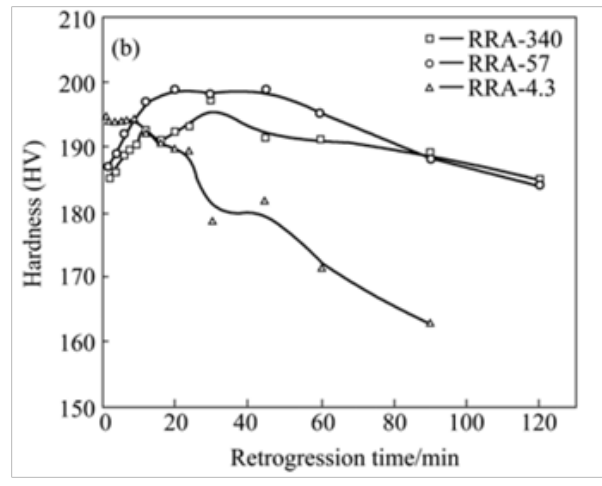

Figure 2 Relationship between retrogression time and resultant hardness.

Decreasing trend of the value of hardness is because of transformation of more $y$ ' into $y$, and coarsening of the $y$ phase. Marlaud et al., ${ }^{30}$ investigated the RRA and PA (peak ageing which is the same as traditional T6 heat treatment) state of the Al-Mg- $\mathrm{Zn}-\mathrm{Cu}$ alloy, and reported that, in both tempers, alloy has the same hardness but different microstructures. It is also stated that, the best time to end the retrogression process is when the size of the precipitates is in the shearing-looping transition state. Another fact that came to light in this research was that, the kinetics of retrogression is very temperature dependent. When the temperature is too high, the number of precipitation which can survive this high temperature is too low, and even at the end of re-aging process no sufficient density of precipitates is resulted. Too low of a retrogression treatment temperature, causes it to be ineffective for the inadequate precipitation dissolution. Songyi Chen et al., ${ }^{31}$ studied the mechanical, corrosion and fracture properties of 7085 aluminum alloy in four different heat treatments: T6, T74, RRA and DRRA (dual RRA). Their research revealed that, while DRRA keeps the strength as high as it is for RRA condition (more than what is for T6 condition) it enhances the SCC resistance as much as T74 heat treatment. It is also reported that, fracture toughness is the same for DRRA and T74 and highest among four. In a research done by LI Jin-feng et al., ${ }^{32}$, effects of two novel heat treatments named HTPP (High Temperature pre precipitation) and T6I6, in addition to those of more common ones; T6, T73 and RRA on the tensile properties, intergranular and exfoliation corrosion and microstructure of 7075 aluminum alloy. The treatment sequence of HTPP is $445^{\circ} \mathrm{C}, 30 \mathrm{~min}+120^{\circ} \mathrm{C}, 24 \mathrm{hr}$ and for T6I6 it is $130{ }^{\circ} \mathrm{C}, 80$ $\min +65{ }^{\circ} \mathrm{C}, 240 \mathrm{hr}+130{ }^{\circ} \mathrm{C}, 18 \mathrm{hr}$. They disseminated the findings of their research through a paper in which it was revealed that, corrosion resistance is increased at the expense of tensile strength. On the other hand, T6I6 and RRA enhanced both tensile strength and corrosion resistance comparing to T6 treatment. Also it is reported that, while $\mathrm{y}$ distribution is continuous in T6 heat treated alloy, it is discontinuous HTPP, T6I6, T73 and RRA conditions. Guosheng Peng et al., ${ }^{33,34}$ studied the impact of repetitious-RRA treatment and dual-RRA on the strength and corrosion resistance of $\mathrm{Al}-\mathrm{Zn}-\mathrm{Mg}-\mathrm{Cu}$ alloy. This investigation showed that, repetitious-RRA made grain boundary precipitates coarser which caused the SCC of the alloy to enhance. Also the size of the precipitates inside the grains changes slightly so hardness of the alloy is not affected much by this heat 
treatment. Regarding the effects of dual-RRA heat treatment, findings show that, dual-RRA treatment, obtains higher exfoliation resistance without losing the strength of RRA heat treated alloy. In another research done by $\mathrm{Yi} \mathrm{Li}$ Wang et al., ${ }^{35}$ it was again proved that, RRA heat treatment has positive influence on the mechanical and corrosion properties of 7050 aluminum alloy. They studied the microstructure and the condition of the precipitates of both inside the grains and grain boundaries using scanning electron microscope (SEM), transmission electron microscope (TEM) and electron backscatter diffraction (EBSD) in the alloy and showed that, inside the grains of RRAed alloy possessed the microstructure similar to that of T6 treated alloy which means the strength is the same in both heat treatments. But the GBPs get coarser after RRA heat treatment which means higher corrosion resistance. Pankade et al., ${ }^{36}$ investigated the effects of RRA and a heat treatment called duplex aging on the mechanical properties and electrical conductivity of 7075 aluminum alloy. Difference between Duplex aging and RRA is that, in duplex aging in contrast to RRA there exist just one heating and cooling cycle. RRA was performed at following conditions: $200^{\circ} \mathrm{C}$ for $2 \mathrm{hr}+120^{\circ} \mathrm{C}$ for $24 \mathrm{hr}$; and duplex aging at $163^{\circ} \mathrm{C}$ for 4 different time durations: $4 \mathrm{hr}, 8 \mathrm{hr}, 16 \mathrm{hr}$ and 24 $\mathrm{hr}$. it was proved that, electrical conductivity improved after all heat treatments in comparison with T6 condition. Duplex treatment with $163^{\circ} \mathrm{C} 16 \mathrm{hr}$ and $24 \mathrm{hr}$ and RRA proved to be most efficient regarding electrical conductivity and corrosion rate; but duplex aging with $163^{\circ} \mathrm{C}$ for $16 \mathrm{hr}$ was the best of all which caused the alloy possess less corrosion rate and more electrical conductivity. Y Liu et al., ${ }^{37}$ applied an special heat treatment called multistage ageing process on 7050 aluminum alloy with the sequence shown in Figure 3 and proved that the variation of hardness and electrical conductivity is affected by heating rate during treatment and that, multistage ageing process with the following sequence: $120^{\circ} \mathrm{C}, 24 \mathrm{hr}+120^{\circ} \mathrm{C}-200^{\circ} \mathrm{C}, 40{ }^{\circ} \mathrm{C} / \mathrm{hr}$ $+120^{\circ} \mathrm{C}, 24 \mathrm{hr}$ possesses the ability to produce a microstructure with strength equivalent to that of T6 treated microstructure and corrosion resistance similar to RRAed alloy.

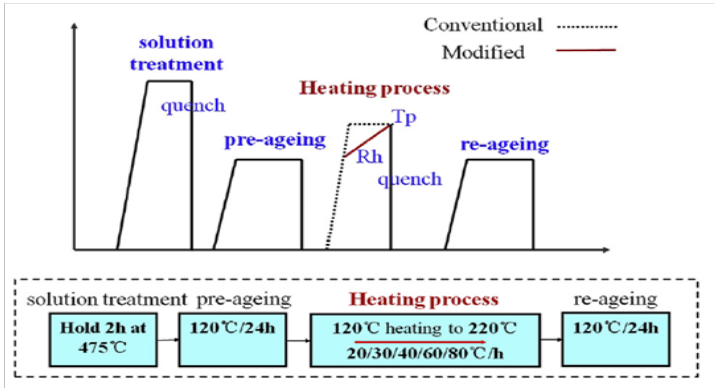

Figure 3 Sequence of multistage ageing process. 37

LL Liu et al., ${ }^{38}$ investigated the effects of various aging treatments such as: T6, RRA and DA (double aging), on the corrosion behavior of 7055 aluminum alloy. The order of corrosion resistance of the resultant microstructures is as follows: DA $>$ RRA $>$ T6. It is stated that, higher corrosion resistance of DA is attributed to the small dispersed $\mathrm{Al}_{7} \mathrm{Cu}_{2} \mathrm{Fe}$; and fine grains along with discontinuous GBPs with causes less electrochemical activity. Thakur et al. ${ }^{39}$ revealed the influence of different ageing treatments on the hydrogen embrittlement (HE) of Al-4.35Zn-1.4Mg-0.059Zr alloy. They showed through TEM studies that, lower dislocation densities of RRAed alloy, HE resistance is improved in it compared to aged samples; and under aged samples possesses more resistance against HE. Effects of three different ageing heat treatments (T6, T73 and RRA) on mechanical behavior of Al-10.8Zn-2.8Mg-1.9Cu alloy was studied by Feng et al., ${ }^{40}$ who showed that, RRA decreased the tensile strength by $\% 3$ comparing with $\mathrm{T} 6$ though the worst mechanical response among three belongs to T73. Lin et al., ${ }^{41}$ investigated the effects of different sorts of heat treatments on the tensile strength (TS) and SCC resistance of 7050 aluminum alloy. In their published paper, it is stated that, T6 treated sample possessed high TS but low SCC resistance; in contrast to T6, T73 caused the sample to show improved SCC but lower TS. On the other hand, although applicable to thin and small specimen, RAA increased both TS and SCC resistance of the alloy.

\section{Suggestions for future researches}

Considering all above-mentioned research and investigations, following suggestions for future research could be made: Finding the optimal values for RRA heat treatment parameters like time and temperature for a given $7 \mathrm{xxx}$ alloy to obtaing the best mechanical and corrosion resistance from the alloy. Relating the percentage of different elements in the chemical composition of alloy to the optimal values of parameters. For example how the time or temperature of the treatment change with changing of percentage of $\mathrm{Zn}$ in an alloy. Defining the effects of different heat treatments on the SPDed (severe plastic deformed) 7xxx alloys.

\section{Conclusion}

An overview of some researches done on the strengthening mechanisms as well as the heat treatment of $7 \mathrm{xxx}$ aluminum alloys is provided in this paper. Dominant strengthening mechanism in AA7075 in ultrafine and coarse grain state was discussed. Also effect of adding $\mathrm{Ag}, \mathrm{Cu}, \mathrm{Zr}, \mathrm{Ni}, \mathrm{Cs}, \mathrm{Fe}$ and cobalt elements to the alloy, on its precipitation and mechanical behavior was clarified. It was shown that adding Ag to the alloy, not only increased the strength after precipitation, but also decreased the precipitation time. And adding $\mathrm{Cu}$ increased the dominating strengthening phase ( $\mathrm{y}$ ') in age hardened $7 \mathrm{xxx}$ alloys and as a result, improved its mechanical strength; zirconium, nickel, cesium, iron and cobalt functioned as grain size stabilizers and prevent grain coarsening. It was shown that aging temperature and quench-induced precipitation play defining role in precipitation and the resultant mechanical strength of the precipitated microstructure. Mechanical properties of the alloy aged from supersaturated condition using electro pulsing method was studied and compared to the traditional supersaturated and aged alloy. An account of double-peak precipitation strengthening was given and it was made clear that, looping is the dominant strengthening mechanism in an alloy treated in such way. The effects of severe plastic deformation methods such as friction stir processing and accumulative roll bounding and high pressure sliding on mechanical behavior and microstructure of 7xxx aluminum alloy was discussed and it was shown that, severe plastic deformation has the capability of causing drastic grain refinement at least in the studied alloys, AA7050 and AA7075 and so boosting the mechanical properties of the alloys. Effect of double step hot rolling on the mechanical properties of AA7055 was discussed and shown that, the process caused the alloy to possess high toughness. It was also stated that, one way of reducing SCC, because of its effect on the dislocations of the surface of the alloy, is shot peening which causes a kind of over-aging to take place in outer layer material which is proved to enhance resistance against SCC. Different aspects of RRA heat treatment, as prominent method of controlling SCC, were discussed. The influence of time, temperature and speed of heating up in retrogression on the microstructural state 
of the alloy were investigated. For AA7075, the temperature and time of retrogression were $180{ }^{\circ} \mathrm{C}$ and 4 mins, respectively to produce the highest hardness. From 3 heating up speeds, the medium one $\left(57^{\circ} \mathrm{C} / \mathrm{min}\right)$ was shown to result in the microstructure with highest mechanical strength. DRRA was proved to have positive impact on both mechanical and corrosion resistance of 7085 aluminum alloy. Among four heat treatments tested on 7075 aluminum alloy, , T6I6 and RRA enhanced both tensile strength and corrosion resistance comparing to $\mathrm{T} 6$ treatment. Also it was shown that, on a given $7 \mathrm{xxx}$ alloy, Dual RRA, has positive influence on exfoliation resistance without losing strength of the alloy. Generally, the review says that, RRA heat treatment is preferable comparing to other ordinary and common heat treatments like T6 and T7x. But there exist other heat treatments like duplex ageing, multistage ageing and double ageing which may have better impact on properties of 7xxx alloys. Regarding the hydrogen embrittlement, it is clear that RRA has positive influence in the alloy.

\section{Acknowledgment}

None.

\section{Conflict of interest}

None.

\section{References}

1. Randolph Kissell RLFJ. Aluminum Structures: A Guide to Their Specifications and Design. 2nd ed. John Wiley \& Sons, Inc; 2002. 544 p.

2. Chen Q, Lin S, Yang C, et al. Grain fragmentation in ultrasonic-assisted TIG weld of pure aluminum. Ultrason Sonochem. 2017;39:403-413.

3. Hatch JE. Aluminum Properties and Physical Metallurgy. ASM International; 1984.

4. Kaufman JG. Introduction to Aluminum Alloys and Tempers. ASM International; 2000.

5. Argon AS. Strengthening Mechanisms in Crystal Plasticity. Oxford University Press; 2008.

6. Whang SH. Nanostructured metals and alloys. Woodhead Publishing, 2011

7. Rouhi S, Mostafapour A, Ashjari M, et al. Effects of welding environmen on microstructure and mechanical properties of friction stir welded AZ91C magnesium alloy joints. Science and Technology of Welding and Joining. 2016;21(1):25-31

8. Ashjari M, Mostafapour Asl A, Rouhi S, et al. Materials Science and Engineering: A. 2015;645:40-46.

9. Ma K, Wen $\mathrm{H}, \mathrm{Hu} \mathrm{T}$, et al. Mechanical behavior and strengthening mechanisms in ultrafine grain precipitation-strengthened aluminum alloy. Acta Materialia. 2014;62(1):141-155.

10. Zhang S, Hu W, Berghammer R, et al. Acta Materialia. 2010;58:66956705.

11. Xu X, Zhao Y, Wang X, et al. Materials Science and Engineering: A 2015;648:367-370.

12. Chen J, Zhen L, Yang S, et al. Investigation of precipitation behavior and related hardening in AA 7055 aluminum alloy. Materials Science and Engineering: A. 2009;500(1-2):34-42.

13. Liu S, Li Q, Lin H, et al. Materials \& Design. 2017;132:119-128.

14. Xu X, Zhao Y, Wang X, et al. Materials Science and Engineering: A. 2016;654:278-281.
15. Nie XW, Zhang LJ, Du Y. Experiments and modeling of double-peak precipitation hardening and strengthening mechanisms in Al-Zn-Mg alloy. Transactions of Nonferrous Metals Society of China. 2014;24(7):21382144 .

16. Yang W, Ding H, Mu Y, et al. Materials Science and Engineering: A. 2017;707:193-198.

17. Alvandi H, Farmanesh K. Microstructural and Mechanical Properties of Nano/Ultra-Fine Structured 7075 Aluminum Alloy by Accumulative RollBonding Process. Procedia Materials Science. 2015;11:17-23.

18. Liao YG, Han XQ, Zeng MX, et al. Materials \& Design . 2015;66:581-586.

19. Zuo J, Hou L, Shi J, et al. Materials Characterization. 2017;130:123-134.

20. Lavernia EJ. Materials Science and Engineering. 1986;211-221.

21. Lee S, Tazo K, Mohamed IF, et al. Materials Science and Engineering: A. 2015;628:56-61.

22. Fooladfar H, Hashemi B, Younesi M, et al. Journal of Materials Engineering and Performance. 2009;19:852-859.

23. Xu DK, Birbilis N, Rometsch PA, et al. Corrosion Science. 2012;54:17-25.

24. Peng GS, Chen KH, Chen SY, et al. Transactions of Nonferrous Metals Society of China. 2012;22:803-809.

25. Oliveira AF, De Barros MC, Cardoso KR, et al. The Effect of RRA on the Strength and SCC Resistance on AA7050 and AA7150 Aluminium Alloys. Materials Science and Engineering: A. 2004;379:321-326.

26. Peng G, Chen K, Chen S, et al. Materials Science and Engineering: A. 2011;528:4014-4018.

27. Xia P, Liu Z, Bai S, et al. Materials Characterization. 2016;118:438-445.

28. Ozer G, Karaaslan A. Transactions of Nonferrous Metals Society of China. 2017;27:2357-2362.

29. Li GF, Zhang XM, Li PH, et al. Transactions of Nonferrous Metals Society of China. 2010;20:935-941.

30. Marlaud T, Deschamps A, Bley F, et al. Acta Materialia. 2010;58:4814 4826.

31. Chen SY, Chen KH, Dong PX, et al. Transactions of Noneferrous Metals Society of China. 2014;24:2320-2325.

32. Feng LJ, Wei PZ, Xing LC, et al. Transactions of Noneferrous Metals Society of China. 2008;18:755-762.

33. Peng G, Chen K, Chen S, et al. Materials Science and Engineering A. 2011;528:4014-4018.

34. Sheng PG, Hua CK, YI CS, et al. Transactions of Noneferrous Metals Society of China. 2012;22:803-809.

35. Wang YL, Pan QL, Wei LL, et al. Materials \& Design. 2014;55:857-863.

36. Pankade SB, Khedekar DS, Gogte CL, et al. The influence of heat treatments on electrical conductivity and corrosion performance of AA 7075-T6 aluminium alloy. Procedia Manufacturing. 2018;20:53-58.

37. Liu Y, Jiang DM, Li WJ, et al. Journal of Alloys and Compounds. 2016;671:408-418.

38. Liu LL, Pan QL, Wang XD, et al. 2017.

39. Thakur A, Raman R, Malhotra SN, et al. Materials Chemistry and Physics. 2007;101:441-447.

40. Feng W, Baiqing X, Yongan Z, et al. Materials Science and Engineering A. 2008;486:648-652.

41. Yongqian X, Lihua Z, Shujian L, et al. Rare Metal Materials and Engineering. 2017;46:0355-0362. 